\title{
C-ANCA Associated Vasculitis Presenting as Isolated Leptomeningeal Enhancement
}

\author{
Marie-Sarah Gagné Brosseau, François Evoy, Sylvie Gosselin
}

Can J Neurol Sci. 2014; 41: 274-277

The anti-neutrophil cytoplasmic antibody (ANCA)-associated vasculitis comprise perinuclear-ANCA (P-ANCA) as well as cytoplasmic-ANCA (C-ANCA). P-ANCA associated vasculitis etiologies are microscopic polyangiitis, allergic angiitis (ChurgStrauss), polyarteritis nodosa and renal-limited vasculitis. The principal C-ANCA related angiitis is granulomatous angiitis (Wegener's granulomatosis) ${ }^{1}$. Central nervous system (CNS) involvement in granulomatous angiitis is uncommon, occurring in only $2 \%-8 \%$ of cases $^{2}$. Meningeal involvement is even more seldom and usually implies the pachymeninges ${ }^{3}$. We report a case of C-ANCA associated vasculitis with focal leptomeningeal enhancement as a unique finding. This is, to our knowledge, the first case described in the literature.

\section{Case Report}

A 63-year-old caucasian man with a long history of extensive atherosclerotic vascular disease presented with a five-month history of multiple stereotyped episodes of left perioral paresthesias spreading to the whole left hemiface and left arm over a few seconds. These episodes lasted between one to five minutes and were sometimes associated with left lower face and left arm weakness. Rarely, there was an associated sensation of mild weakness in the left leg. The patient denied any relation between the symptoms and posture changing. None of the episodes included clonic movements, visual symptoms, language difficulties, headache, dizziness or altered consciousness. The frequency was one episode every one or two days. He had consulted his family doctor about three months earlier for the same episodes and Clopidogrel 75mg per os once daily was added to Aspirin $80 \mathrm{mg}$ he was already taking since 1999, but the patient did not notice any improvement. Neither at presentation nor in the past had he had upper or lower respiratory tract complaints suggestive of vasculitis, chronic sinusitis, hematuria, arthralgia and rash. He had a history of bilateral carotid endarterectomy in 1999 for transient ischemic attacks and right commun carotid artery stenting in 2001 for recurrent transient ischemic attack associated with restenosis at the site of previous endarterectomy. He didn't have any neurological deficit. He also had renal angioplasty in 1999 and 2001. His other health conditions included high blood pressure, dyslipidaemia and coronary artery disease. The patient had a 50 pack-years smoking history and was still actively smoking. He denied alcohol or drugs consumption. On examination, the vital signs were within normal limits. There was a right carotid murmur. General physical examination was completly normal. Neurologic examination showed mild weakness of left arm, a

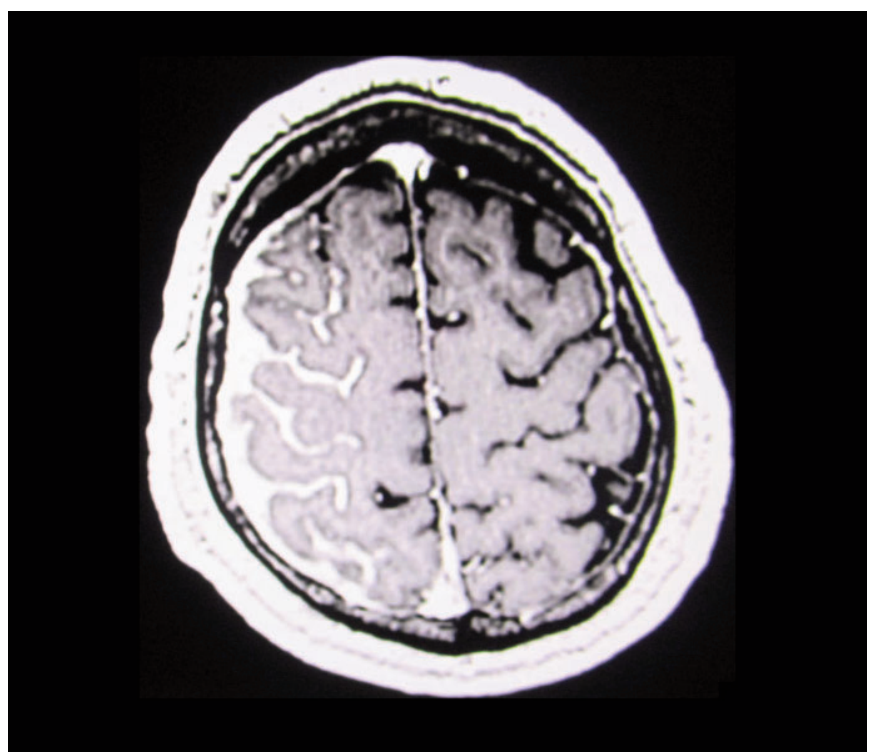

Figure 1: T1W with Gadolinium injection

slightly positive pronator drift test on the left side as well as dysdiadochokinesia of left finger-hand movements and increased deep tendon reflexes on the left side. The rest of the neurological examination was normal.

The head computed tomography (CT) without contrast revealed right frontoparietal hyperdensity of the leptomeninges. The cortex seemed normal. The head CT done three months earlier was reviewed and showed similar but very subtle findings in the same location. A carotid angioscan demonstrated less than $50 \%$ stenosis of the right common carotid artery stent and subocclusive stenosis of the left internal carotid artery. Brain magnetic resonance imaging (MRI) revealed hyperintense signals on fluid attenuated inversion recovery (FLAIR) and T2weighted (T2W) ponderations of the right frontoparietal

From the Centre Hospitalier Universitaire de Sherbrooke, Division of Neurology, Sherbrooke University, Sherbrooke, Québec, Canada

Received September 18, 2013. Final Revisions Submitted October 7, 2013. Correspondence to: Marie-Sarah Gagné Brosseau, Centre Hospitalier Universitaire de Sherbrooke, 3001 12th Avenue North, Sherbrooke (Quebec), J1H 5N4, Canada. Email: marie-sarah.gagne.brosseau@usherbrooke.ca. 


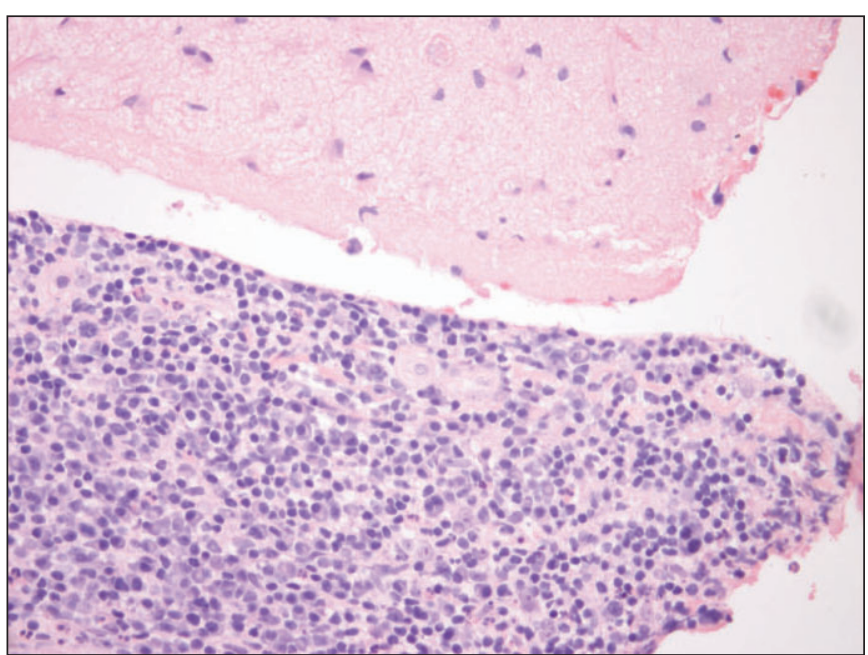

Figure 2: $H \& E$ stain showing infiltrate of the leptomeninges sparing the cortex (zoom 40x)

leptomeninges, which enhanced after contrast injection (Figure 1 ). There was subtle hyperintensity of the adjacent cortex on FLAIR and T2W but no area of restricted diffusion or evidence of blood. The paranasal sinuses and bones were unremarkable. A pancorporal positron emission tomography (PET) scan showed hypermetabolism in the right frontoparietal region measuring 9 X 5,8 X 0,8 centimeters and was otherwise normal.

Laboratory tests were analysed: urine analysis, electrolytes as well as liver, thyroid and kidney function tests were all normal. Complete blood count revealed slighly elevated absolute eosinophil number of 0,6 per liter (normal value $0-0,2$ ), which had been present on past complete blood count (CBC)s for the last 20 years. Sedimentation rate was of $60 \mathrm{~mm} / \mathrm{h}$ (normal range: $0 \pm 20 \mathrm{~m} / \mathrm{h}$ ). Angiotensin-converting enzyme, beta-2microglobulines, serum protein electrophoresis and immunofixation were normal just as C3 and C4 levels. Human

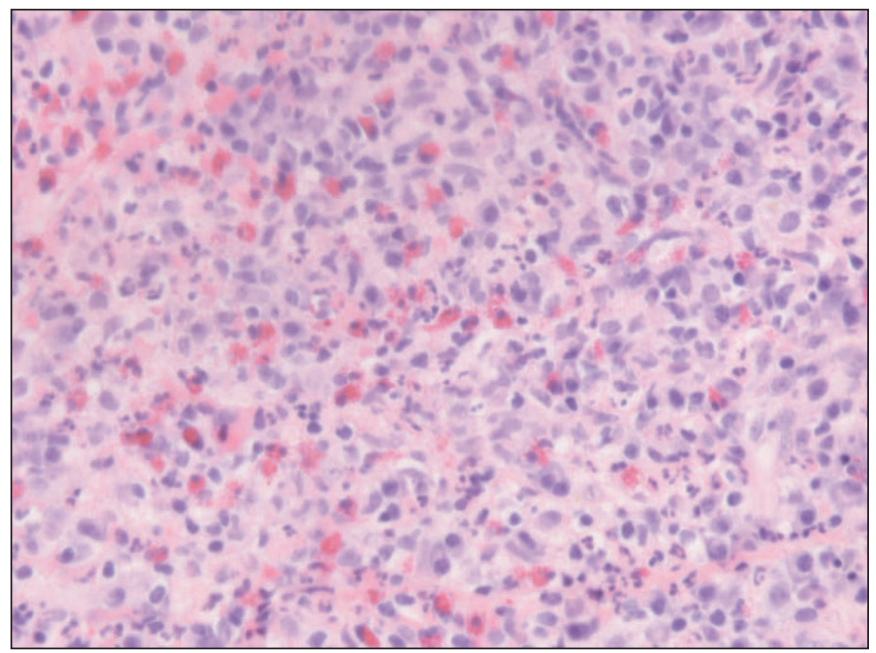

Figure 4: H\&E stain showing a few scattered mastocytes (zoom 60x)

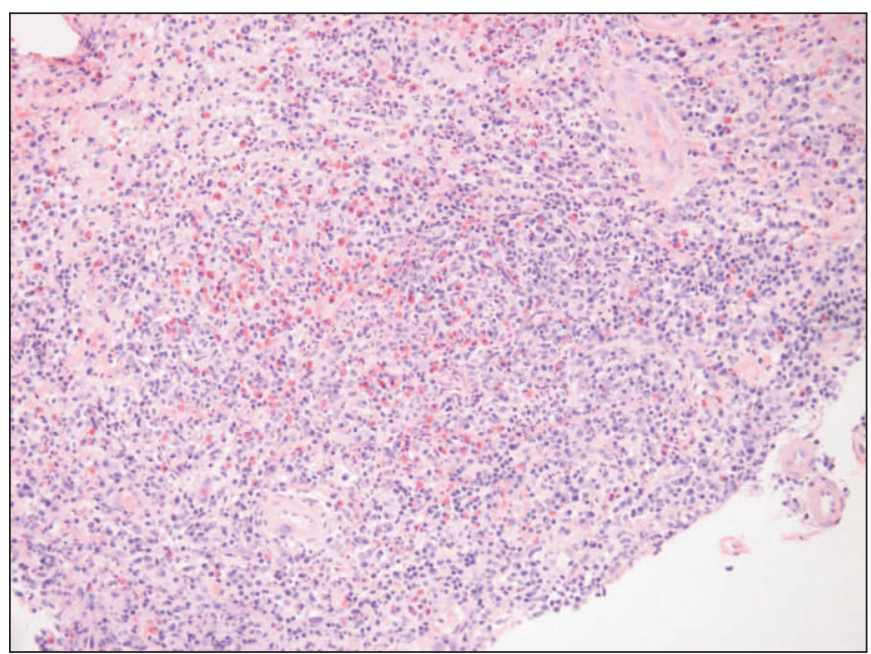

Figure 3: $H \& E$ stain showing meningeal lymphocytic infiltrate infiltrate (zoom 20x)

immunovirus (HIV), rheumatoid factor and antinuclear antibody were negative. Serum C-ANCAs were strongly positive, with a value of 1:320 and anti-PR3 antibodies were within the normal range. ANCAs were not tested on CSF. The purified protein derivative (PPD) skin test was negative. Cerebrospinal fluid studies revealed normal glucose $(3,5 \mathrm{mmol} / \mathrm{L})$ and slightly increased proteins to $0,82 \mathrm{mmol} / \mathrm{L}$ (normal value $0,2-0,6$ ). The cell count enumerated 26 erythrocytes $/ \mathrm{mm}^{3}$ and 17 leukocytes $/ \mathrm{mm}^{3}$ (100\% mononuclear). Cytology identified lymphocytes in slight excess compared to normal with benign appearance as well as rare neutrophils and monocytes. Cultures for bacteries, mycobacteries, virus, parasites and mycosis were all negative. Finally, beta-2-microglobulin and angiotensinconverting enzyme levels were within normal limits.

Meningeal biopsy of the right frontal lobe (Figures 2-4) showed a perivascular infiltrate containing abundant $\mathrm{T}$

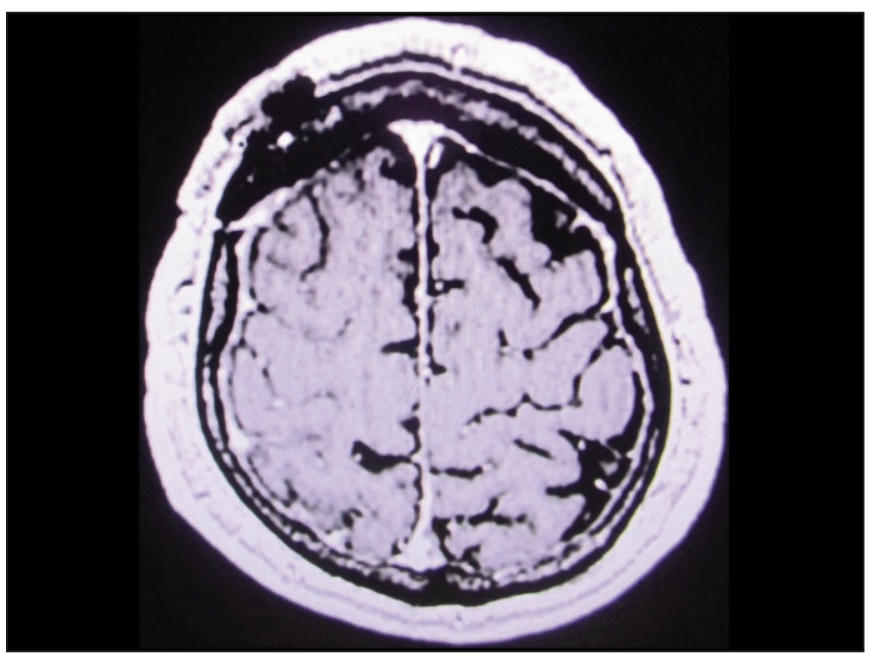

Figure 5: T1W with Gadolinium injection 
Table 1: Various etiologies of leptomeningeal enhancement

\begin{tabular}{l|l}
\hline Diffuse & Focal \\
\hline -Carcinomatous or & -Carcinomatous or lymphomatous \\
lymphomatous & meningitis \\
meningitis & -Primary brain tumor spreading \\
-Primary brain tumor spreading & -Post-convulsion \\
-Subacute state of subarachnoid & -Infectious meningitis (ex: \\
hemorrhage & neurosyphilis, tuberculous) \\
-Infectious meningitis (ex: & -Subacute state of ischemic stroke \\
bacterial, viral, tuberculous, & -Inflammatory (ex: collagen \\
fungic, mycotic and Whipple's & vascular disease, rheumatoid \\
disease) & arthritis and neusarcoidosis) \\
-Inflammatory (ex: & -Infusion of hyperosmolar solution \\
neurosarcoidosis) & -Sturge-Weber disease \\
-Chemical meningitis (ex: & \\
cysticercoma or dermoid cyst & \\
rupture) & \\
\hline
\end{tabular}

lymphocytes and a few scattered mastocytes in the leptomeninges, sparing the pachymeninges and the cortex, compatible with leptomeningeal vasculitis. The cultures for bacteries, mycobacteries, virus, mycosis, amibes and other parasites all were negative. Special stains for acid-fast bacilli, fungi, bacteria, and spirochetes were negative as well.

Chest CT revealed patchy ground-glass opacities and centrilobular emphysema consistent with smoking-related interstitial lung disease. There was also scarce reticular pattern due to fibrosis. Two electroencephalograms (EEG) performed during hospital stay were normal. Despite the normal EEGs, a diagnosis of simple partial seizures was established so Levetiracetam 500mg per os twice daily was intruduced. After the beginning of antiepileptic medication, the episodes gradually waned over a period of two weeks.

Once neoplastic and infectious causes were ruled out, the most probable diagnosis remaining was focal C-ANCAassociated angiitis. Prednisone 50mg per os once daily was started. Control cerebral MRIs, made four weeks and six months later, both revealed $95 \%$ reduction of the right leptomeningeal abnormalities, resolution of the adjacent cortical FLAIR hyperintensity and no new enhancing lesion (Figure 5). The control C-ANCA value had normalised and the anti-MPO antibodies (tested a few weeks after initiation of Prednisone) were also negative.

Moreover, the paroxystic episodes have not recurred since the treatment was established and the neurological examination has normalized. After two months with the same dosing of Prednisone, it has been slowly reduced over six weeks to a dose of $20 \mathrm{mg}$ daily and Methotrexate was added.

\section{Discussion}

Leptomeningeal enhancement, which represents enhancement of the pia mater or subarachnoid spaces of the sulci and cisterns, is a nonspecific finding with many possible causes. It can be classified as diffuse of focal. The various etiologies are
Table 2: Various etiologies of pachymeningeal enhancement

\begin{tabular}{l|l}
\hline Diffuse & Focal \\
\hline -Idiopathic hypertrophic & -Metastases (ex: lymphoma, \\
pachymeningitis & craniofacial neoplasms, breast \\
-CSF leak (ex: shunting, & carcinoma, prostate carcinoma, \\
post-lumbar puncture, & melanoma) \\
spontaneous intracranial & -Extra-axial neoplasm (ex: \\
hypotension) & meningioma) \\
-Post-radiotherapy & -Infectious (ex: bacterial, \\
-Post-trauma & tuberculosis and fungic) \\
-Inflammatory (ex: & -Inflammatory (ex: \\
Wegener's granulomatosis, & neurosarcoidosis, Wegener's \\
neurosarcoidosis) & gynulomatosis, Behçet's \\
-Chemical meningitis (intra- & Vogt-Koyanagi-Harada disease) \\
thecal chemotherapy, & -Reactive (post-craniotomy) \\
cysticercoma or dermoid cyst & \\
rupture) & \\
-IgG4-related syndrome & \\
\hline
\end{tabular}

represented in Table 1 and are different from those of pachymeningeal enhancement, as presented in Table $2^{4}$.

Granulomatous angiitis (or Wegener's granulomatosis (WG)) is a rare autoimmune disorder characterized by acute necrotizing granulomatous lesions of the upper and lower respiratory tract, multifocal necrotizing vasculitis affecting the small arteries and veins of the respiratory tract and other sites, and focal or diffuse glomerulonephritis ${ }^{5}$. It may begin with a subclinical phase, which is sometimes prolonged. In approximately $20 \%$ of patients it may present as a limited form 6 .

The gold standard for diagnosis is biopsy demonstrating typical findings, but the sensitivity is not so high. In a study evaluating 126 head and neck biopsy specimens performed in 70 patients affected by WG, vasculitis was shown in $26 \%$, necrosis in $33 \%$ and granulomatous inflammation in 42\%; the combination of these three features was seen only in $16 \%$ of the cases $^{7}$. This probably explains why our biopsy didn't reveal granulomas or necrosis.

The nervous system is frequently affected (22-54\% of cases), with the condition most commonly manifesting as mononeuritis multiplex secondary to vasculitis of the vasa nervorum. However, CNS involvement is uncommon, occurring in only $2 \%-8 \%$ of patients ${ }^{2}$. Symptoms and signs are nonspecific and may be isolated headache, cranial nerve palsies, pituitary involvement, confusion/coma or focal brain lesion. The patterns of affection vary widely and can be classified as follows: contiguous invasion from extracranial sites (paranasal sinuses, periauricular tissues), remote intracranial granuloma, CNS vasculitis and meningeal involvement. ${ }^{8}$

Meningeal involvement may be diffuse or focal and usually presents with dural thickening and enhancement sometimes compatible with hypertrophic pachymeningitis (HP). Leptomeningeal involvement is even more unusual. In the 
literature (PubMed reviewed on July 30th 2013), only two cases of leptomeningeal involvement ${ }^{9,10}$ and two cases of combined pachymeningeal and leptomeningeal involvement ${ }^{6,11}$ were reported. None of these patients presented with isolated CNS involvement.

Most patients with meningeal involvement previously described in the literature had mild disease limited to the respiratory tract ${ }^{9}$. Nervous system insult is generally a late finding, but may be inaugural in rare cases ${ }^{8}$.

An immunofluorescent pattern of C-ANCA has a sensitivity of at least $90 \%$ and a specificity approaching $99 \%$ for diagnosing WG. Among WG patients with positive C-ANCA, 80-90\% have PR3-ANCA and the remaining mainly MPO-ANCA or bactericidal permeability increasing $(\mathrm{BPI})^{6}$. Differently from anti-PR3 antibodies, which are highly specific for WG, antiMPO antibodies have been found in a larger group of vasculitic and nonvasculitic disorders ${ }^{1}$. In our case, the C-ANCAs were strongly positive (1:320) but the anti-PR3 antibodies were negative.

Among ANCA-associated vasculitis, the positivity of antiMPO antibodies is associated with a lower vasculitic activity index, a lesser number of involved organs and a lower rate of relapse. Moreover, MPO-ANCA vasculitis could belong to the limited form of Wegener's granulomatosis and lack the typical clinical triad ${ }^{12}$. In our patient, the involvement limited to central nervous sytem and the negative anti-PR3 antibodies correlate with these observations.

Finally, in the present case, because the eosinophilia was very mild and there never had been any eosinophil visualized on biopsy and CSF cytology, Churg-Strauss was ruled out.

In conclusion, to the best of our knowledge, this is the first case report in the literature of C-ANCA related isolated focal leptomeningeal inflammation.

\section{REFERENCES}

1. Van der Woude FJ, Rasmussen N, Lobatto S, et al. Autoantibodies against neutrophils and monocytes: tool for diagnosis and marker of disease activity in Wegener's granulomatosis. Lancet. 1985;1:425-29.

2. Nishino H, Rubino FA, DeRemee RA, Swanson JW, Parisi JE. Neurological involvement in Wegener's granulomatosis: an analysis of 324 consecutive patients at the Mayo Clinic. Ann Neurol. 1993;33:4-9.

3. Tasali N, Cubuk R, Celik L, Canbora B. Atypical meningeal involvement in Wegener granulomatosis: MR imaging survey of a particular case. Am J Neuroradiol. 2009;30:E116.

4. Smirniotopoulos JG, Murphy FM, Rushing EJ, Rees JH, Schroeder JW. Patterns of contrast enhancement in the brain and meninges. Radiographics. 2007;27:525-51.

5. Albayram S, Kizilkilie O, Adaletli I, Erdogan N, Kocer N, Islak C. MR imaging findings of spinal dural involvement with Wegener granulomatosis. Am J Neuroradiol. 2002;23:1603-6.

6. Nusbaum AO, Morgello S, Atlas SW. Pial involvement in Wegener's granulomatosis shown on MRI. Neuroradiology. 1999;41:847-9.

7. Caramaschi P, Biasi D, Carletto A, Bambara LM. A case of ANCAassociated vasculitis with predominant involvement of central nervous system. Joint, bone, spine. 2003;70:380-3.

8. Ossi E, Rossanese A, Casagrande F, Rotilio A, Mingrino S, Poci C. Predictive value of ANCA in atypical primary brain localization of Wegener's granulomatosis. Sarcoidosis Vasc Dif. 2002;19: 78-80.

9. Di Comite G, Bozzolo E, Bianchi S, Sabbadini MG. Two cases of meningeal involvement in Wegener's granulomatosis. Rheumatology. 2004;43:1459-60.

10. Yildirim N, Arslanoglu A, Aygun N. Otologic and leptomeningeal involvements as presenting features in seronegative Wegener granulomatosis. Am J Otolaryng. 2008;29:147-9.

11. Al Dhanhani A, Macaulay R, Maloney B, Hanly JG. Meningeal involvement in Wegener's granulomatosis. J Rheumatol. 2006; 33:364-7.

12. Horino $\mathrm{T}$, Takao $\mathrm{T}$, Taniguchi $\mathrm{Y}$, Terada $\mathrm{Y}$. Hypertrophic pachymeningitis with MPO-ANCA-positive vasculitis. Clin Rheumatol. 2010;29:111-13. 